\title{
A Study on Diversity of Spiders at Malavagoppa Village, in Shimoga District, Karnataka
}

\author{
Shraddha Kumari $\mathrm{K}^{1}$, Chaturved Shet $\mathrm{R}^{2}$ \\ ${ }^{1}$ Department of Applied Zoology, Kuvempu University, Shankaraghatta, Shimoga. \\ Email: shraddhakkumari@gmail.com \\ ${ }^{2}$ Reseracher and Co-founder, Harish R Bhat Science Foundation, Yeshwanthpur, Bangalore. \\ Email: chaturvedshet@gmail.com
}

\begin{abstract}
Spiders belong to the order Araneae which is the largest order among the class Arachnida. Spiders are considered as indicators of overall species richness and the health of terrestrial communities. The village with different plantations harbors a good number of spiders. The survey was carried out from February 2018 to January 2019 by using active searching, beating, leaf sampling and net sweeping techniques. During the study, a total number of 51 species belonging to 42 genera of 16 families were documented. In the present study it was found that the family Araneidae was dominated by having 14 species followed by the family Salticidae having 12 species and the least were from Clubionidae, Corinnidae, Eresidae, Gnaphosidae, Hersiliidae, Linyphiidae, Sparassidae, Theridiidae and Uloboridae with a single species from each family.
\end{abstract}

Keywords-Araneidae, Malavagoppa Village, Spiders Diversity.

\section{INTRODUCTION}

Spiders form one of the most ubiquitous groups of predaceous organisms in the animal kingdom (Riechert and Lockley 1984). Among all organisms, spiders (Order: Araneae) form the seventh largest order in terms of number of known species (Sebastian and Peter, 2009). They belong to the class Arachnida of the Phylum Arthropoda that possesses jointed appendages with a chitinous exoskeleton system. They are characterized by two body parts, the cephalothorax having 4 pairs of segmented legs and the abdomen. They have simple eyes, no antenna and no wings, which differentiate them from insects.

Spiders are the key components of all ecosystems in which they live. They are the ancient animals with a history going back over 350 million years (Sebestian and Peter, 2010). This is the most diverse, female dominated and entirely predatory order in the Arthropod world. Globally, spiders include about 48,147 described species in 4131 genera and 117 families. (World Spider Catalog, 2019). They are distributed on every continent except
Antarctica and have adapted to all known ecological environments except air and the open sea (Foelix, 1996). Spiders of the Western Ghats are poorly studied compared to otherparts of the country. With respect to its geographical, climatic and ecological features, the Western Ghats harbors a rich amount of arachnids out of which spiders have a huge share. Studies on Indian spiders were initiated by Blackwall (1850). Different studies have shown that spiders regulate prey populations depending on their density. Recent studies have shown that the spiders act as ecological indicators and early warning signs of environmental changes (Kremen et. al., 1993).

\section{MATERIALS AND METHOD}

\section{Study site:}

Malavagoppa village, located at $13.8998^{\circ} \mathrm{N} 75.6194^{\circ} \mathrm{E}$ Shimoga district, Karnataka. This village is an urban area with good number of vegetation.

\section{Methods:}

Study sites included indoor habitats such as inside houses and other buildings, surroundings of human dwellings; outdoor habitats included were gardens, areca plantations, and open field.

The study was carried out from February 2018 to January 2019 covering all three seasons.

Survey was done 2 times in a week. The techniques involved were all out active searching, beating, visual observation and photographic methods. Collections were done by hand picking, gentle beating on surroundings so as to make the individual pass into the collection box. Detailed morphological study was done using Stereozoom and compound microscope. Identifications were done by referring 'Spiders of India' book, literatures and other scientific papers.

\section{Statistical analysis:}

Calculations were done by using Microsoft excel.

Formulae used:

$$
\text { Frequency }=\frac{\text { Number of seasons studied }}{\text { Total number of seasons studied }}
$$


Density $=\frac{\text { Total number of individuals of a species }}{\text { Number of seasons studied }}$

Abundance

Total number of individuals of a species

$=\frac{\text { Number of seasons in which occured } 2 a}{\text { Total }}$

Shannon's diversity index, $\mathrm{H}=-\sum \mathrm{Pi}(\ln \mathrm{Pi})$

Simpson's value, $\mathrm{D}=\sum \mathrm{ni}(\mathrm{ni}-1) / \mathrm{N}(\mathrm{N}-1)$

Where, $n i=$ Number of individuals of a species

$\mathrm{N}=$ Total number of all individuals

\section{RESULTS}

A. Species diversity of spiders

The study site has a great spider diversity of 51 species belonging to 42 genera of 16 families.

In the present study site, the family Araneidae is dominated by having 14 species (9 genera) followed by the family Salticidae having 12 species ( 12 genera), the Tetragnathidae with 6 species (3 genera), Lycosidae with 3 species ( 3 genera), Oxyopidae with 3 species ( 2 genera), Thomisidae and Pholcidae with 2 species ( 2 genera) in each and the Clubionidae, Corinnidae, Eresidae, Gnaphosidae, Hersiliidae, Linyphiidae, Sparassidae, Theridiidae and Uloboridae were identified with single species in each (Table.1).

Table.1: Spiders recorded in Malavagoppa village during Feb 2018-Jan 2019

\begin{tabular}{|c|c|c|c|c|}
\hline Sl. No & Family & Scientific name & Common name & Species count \\
\hline \multirow{14}{*}{1} & \multirow{14}{*}{ Araneidae } & Araneus spp (Clerck, 1757) & Angulated Orb Weaver & \multirow{14}{*}{14} \\
\hline & & Argiope anasuja (Throell,1887) & Signature Spider & \\
\hline & & Argiope pulchella (Throell,1881) & Garden Cross Spider & \\
\hline & & Argiope spp.1 (Audouin, 1826) & Signature Spider & \\
\hline & & Cyclosa bifida (Doleschall, 1859) & Long-bellied Cyclosa & \\
\hline & & Cyrtophora cicatrosa (Stoliczka, 1869) & Tent-web Spider & \\
\hline & & Cyrtophora citricola (Forsskal, 1775) & Tropical Tent-web Spider & \\
\hline & & Eriovixia spp.(Archer, 1951) & & \\
\hline & & $\begin{array}{l}\text { Gasteracantha geminata (Fabricius, } \\
1798 \text { ) }\end{array}$ & Oriental Spiny Orb-weaver & \\
\hline & & Gasteracantha spp.1 (Sundevall, 1833) & Spiny Orb-weaver & \\
\hline & & Neoscona crucifera (Lucas, 1838) & Common Garden Spider & \\
\hline & & Neoscona nautica (L. Koch, 1875) & Grey Sphere Spider & \\
\hline & & Parawixia dehaani (Doleschall, 1859) & Abandoned Orb-weaver & \\
\hline & & $\begin{array}{l}\text { Thelacantha brevispina (Doleschall, } \\
\text { 1857) }\end{array}$ & $\begin{array}{l}\text { False Gasteracantha } \\
\text { (Double Spotted Spiny Spider) }\end{array}$ & \\
\hline 2 & Clubionidae & Clubiona spp. (Latreille, 1804) & Leaf Curling Sac Spiders & 1 \\
\hline 3 & Corinnidae & Castianeira zetes (Simon, 1897) & Black Ant-mimicking Spider & 1 \\
\hline 4 & Eresidae & Stegodyphus sarasinorum (Karsch,1892) & Indian Social Spider & 1 \\
\hline 5 & Gnaphosidae & Gnaphosa spp. (Latreille, 1804) & Ground Dwelling Spider & 1 \\
\hline 6 & Hersiliidae & Hersilia savignyi (Lucas, 1836) & Two Tailed Spider & 1 \\
\hline 7 & Linyphiidae & Neriene sundaica (Simon, 1905) & Black Dwarf Spider & 1 \\
\hline \multirow{3}{*}{8} & \multirow{3}{*}{ Lycosidae } & Geolycosa spp.(Montgomery, 1904) & Wolf spider & \multirow{3}{*}{3} \\
\hline & & Hippasa agelenoides(Simon,1884) & Funnel Web Spider & \\
\hline & & Pardosa spp. (C. L. Koch,1847) & Wolf Spider & \\
\hline \multirow{3}{*}{9} & \multirow{3}{*}{ Oxyopidae } & Oxyopes javanus (Throell,1887) & Striped Lynx Spider & \multirow{3}{*}{3} \\
\hline & & Oxyopes sunandae (Tikader, 1970) & Orange Lynx Spider & \\
\hline & & Peucetia viridians (Hentz,1832) & Green Lynx Spider & \\
\hline \multirow{2}{*}{10} & \multirow{2}{*}{ Pholcidae } & Crossopriza lyoni (Blackwall, 1867) & Tailed Cellar Spider & \multirow{2}{*}{2} \\
\hline & & Pholcus spp. (Walckenaer, 1805) & Daddy long leg spider & \\
\hline \multirow{3}{*}{11} & \multirow{3}{*}{ Salticidae } & $\begin{array}{l}\text { Asemonea spp. (O. Pickard- } \\
\text { Cambridge,1869) }\end{array}$ & Green jumping spider & \multirow{3}{*}{12} \\
\hline & & Bavia kairali (Samson \& Sebastian,2002) & Scorpion Spider & \\
\hline & & Carrhotus viduus (C. L. Koch,1847) & Double Striped Carrhotus & \\
\hline
\end{tabular}




\begin{tabular}{|c|c|c|c|c|}
\hline & & Chrysilla volupe (Karsch,1879) & Colored Jumping Spider & \\
\hline & & Epeus indicus (Proszynski,1992) & White Spotted Green Jumper & \\
\hline & & Hyllus semicupreus (Simon, 1885) & Heavy-bodied Jumping Spider & \\
\hline & & Menemerus bivittatus (Dufour, 1831) & Gray Wall Jumper & \\
\hline & & $\begin{array}{l}\text { Myrmarachne plataleoides (O. Pickard- } \\
\text { Cambridge,1869) }\end{array}$ & Kerengga Ant-like Jumper & \\
\hline & & Phintella vittata (C. L. Koch,1846) & Banded Phintella & \\
\hline & & Plexippus paykulli (Audouin, 1826) & Pantropical Jumping Spider & \\
\hline & & Portia albimana (Simon, 1900) & Fringed Jumping Spider & \\
\hline & & Telamonia dimidiata (Simon, 1899) & Two-striped Jumping Spider & \\
\hline 12 & Sparassidae & Heteropoda venatoria (Linnaeus, 1767) & Huntsman Spider & 1 \\
\hline \multirow{6}{*}{13} & \multirow{6}{*}{ Tetragnathidae } & Leucauge decorata (Blackwall, 1864) & Decorative silver orb weaver & \multirow{6}{*}{6} \\
\hline & & Leucauge tessellata (Throell,1887) & Long jawed orb weaver & \\
\hline & & Leucauge spp.1 (White, 1841) & Decorative spider & \\
\hline & & Opadometa fastigata (Simon, 1877) & Pear Shaped Leucauge & \\
\hline & & $\begin{array}{l}\text { Tetragnatha mandibulata (Walckenaer, } \\
1841 \text { ) }\end{array}$ & Long-jawed Orb-weaver & \\
\hline & & Tetragnatha spp.1 (Latreille, 1804) & Long jawed Spider & \\
\hline 14 & Theridiidae & Theridion spp. (Walckenaer, 1805) & Comb-footed Spider & 1 \\
\hline \multirow[t]{2}{*}{15} & \multirow[t]{2}{*}{ Thomisidae } & $\begin{array}{l}\text { Misumenops rubrodecoratus } \\
\text { (Millot,1942) }\end{array}$ & Green Crab Spider & \multirow[t]{2}{*}{2} \\
\hline & & Thomisus spectabilis (Doleschall, 1859) & White Crab spider & \\
\hline 16 & Uloboridae & Uloborus spp. (Latreille, 1806) & Feather-footed Spider & 1 \\
\hline
\end{tabular}

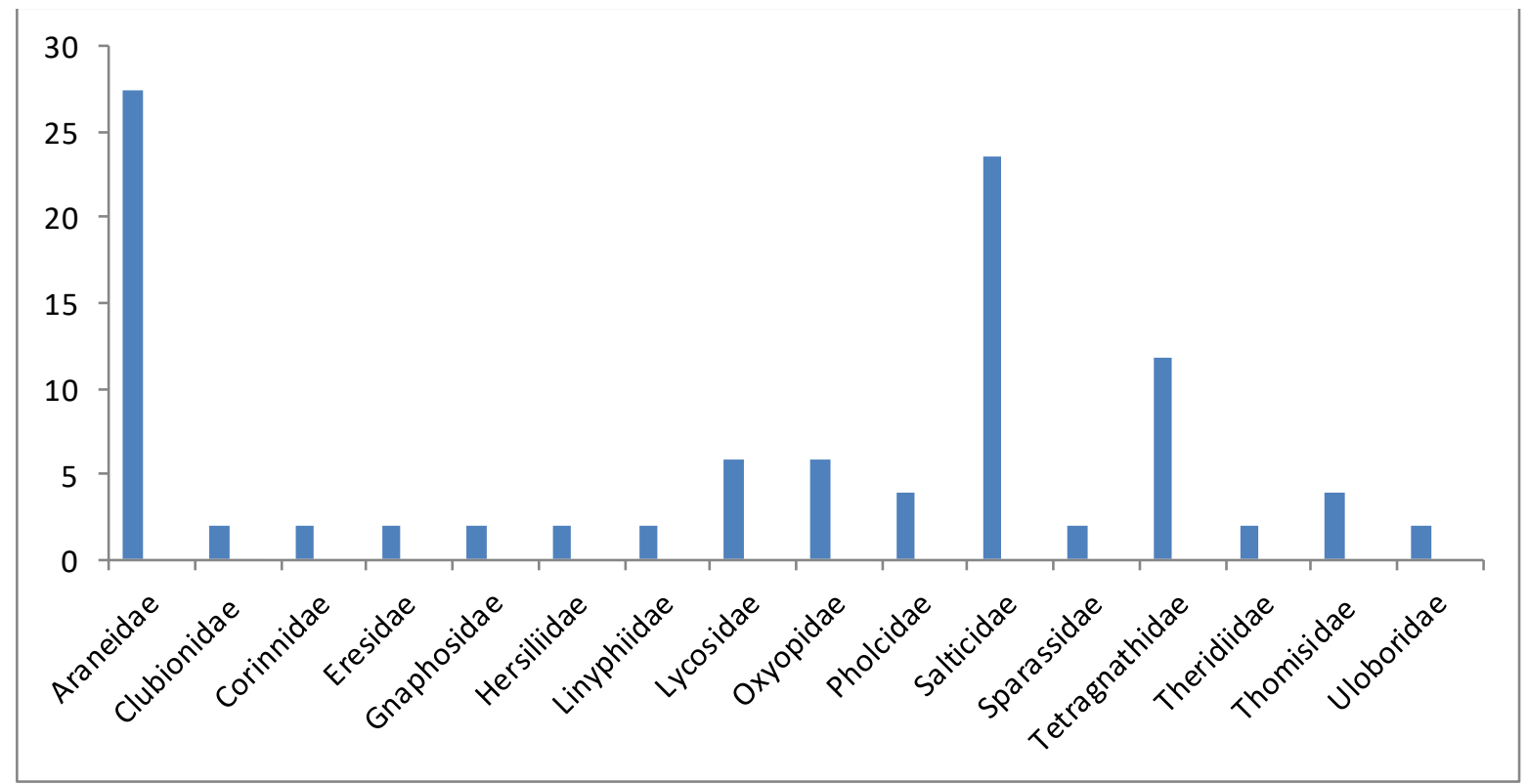

Fig.1: Graph of species percentage under each family

\section{B. Diversity richness and seasonal abundance}

Three main seasons were studied viz., rainy, winter and summer. Most spider species were found throughout the study period and few were found only in two seasons. But, only one species (Oxyopes sunandae) has occurred in one season (rainy). (Table 2). 
Table.2. Shows individual counts of each species during different seasons.

\begin{tabular}{|c|c|c|c|c|c|}
\hline \multirow[t]{2}{*}{ SI No. } & \multirow[t]{2}{*}{ Spider Species } & \multicolumn{3}{|c|}{$\begin{array}{c}\text { Individual counts of spider in } \\
\text { different seasons } \\
\end{array}$} & \multirow[t]{2}{*}{ Total count } \\
\hline & & Rainy & Winter & Summer & \\
\hline 1 & Araneus spp & 11 & 9 & 3 & 23 \\
\hline 2 & Argiope anasuja & 12 & 13 & 9 & 34 \\
\hline 3 & Argiope pulchella & 13 & 14 & 8 & 36 \\
\hline 4 & Argiope spp.1 & 6 & 5 & 3 & 14 \\
\hline 5 & Asemonea spp & 8 & 9 & 6 & 23 \\
\hline 6 & Bavia kairali & 2 & 1 & 0 & 3 \\
\hline 7 & Carrhotus viduus & 4 & 4 & 3 & 11 \\
\hline 8 & Castianeira zetes & 15 & 16 & 11 & 42 \\
\hline 9 & Chrysilla volupe & 12 & 13 & 16 & 41 \\
\hline 10 & Clubiona spp & 14 & 8 & 6 & 28 \\
\hline 11 & Crossopriza lyoni & 50 & 55 & 50 & 155 \\
\hline 12 & Cyclosa bifida & 25 & 23 & 15 & 63 \\
\hline 13 & Cyrtophora cicatrosa & 40 & 44 & 25 & 109 \\
\hline 14 & Cyrtophora citricola & 20 & 20 & 12 & 52 \\
\hline 15 & Epeus indicus & 4 & 4 & 3 & 11 \\
\hline 16 & Eriovixia spp. & 4 & 3 & 3 & 10 \\
\hline 17 & Gasteracantha geminata & 12 & 12 & 0 & 24 \\
\hline 18 & Gasteracantha spp.1 & 2 & 1 & 0 & 3 \\
\hline 19 & Geolycosa spp. & 40 & 45 & 50 & 135 \\
\hline 20 & Gnaphosa spp & 8 & 7 & 2 & 17 \\
\hline 21 & Heteropoda venatoria & 6 & 4 & 4 & 14 \\
\hline 22 & Hersilia savignyi & 10 & 11 & 11 & 32 \\
\hline 23 & Hippasa agelenoides & 30 & 31 & 29 & 90 \\
\hline 24 & Hyllus semicupreus & 4 & 3 & 2 & 9 \\
\hline 25 & Leucauge decorata & 12 & 15 & 7 & 34 \\
\hline 26 & Leucauge tesselata & 4 & 2 & 0 & 6 \\
\hline 27 & Leucauge spp.1 & 2 & 1 & 0 & 3 \\
\hline 28 & Menemerus bivittatus & 10 & 8 & 9 & 27 \\
\hline 29 & Misumenops rubrodecoratus & 1 & 2 & 0 & 3 \\
\hline 30 & Myrmarachne plataleoides & 16 & 17 & 15 & 48 \\
\hline 31 & Neoscona crucifera & 32 & 34 & 33 & 81 \\
\hline 32 & Neoscona nautica & 4 & 3 & 2 & 9 \\
\hline 33 & Neriene sundaica & 30 & 34 & 15 & 79 \\
\hline 34 & Oxyopes javanus & 45 & 50 & 50 & 145 \\
\hline 35 & Oxyopes sunandae & 2 & 0 & 0 & 2 \\
\hline 36 & Opadometa fastigata & 2 & 2 & 0 & 4 \\
\hline 37 & Parawixia dehaani & 1 & 1 & 0 & 2 \\
\hline 38 & Pardosa spp & 45 & 56 & 60 & 161 \\
\hline 39 & Peucetia viridans & 20 & 25 & 16 & 61 \\
\hline 40 & Phintella vittata & 2 & 4 & 8 & 14 \\
\hline 41 & Pholcus spp & 55 & 50 & 55 & 160 \\
\hline 42 & Plexippus paykulli & 62 & 65 & 64 & 191 \\
\hline
\end{tabular}




\begin{tabular}{|l|l|r|r|r|r|}
\hline 43 & Portia albimana & 0 & 2 & 2 & 4 \\
\hline 44 & Stegodyphus sarasinorum & 7 & 8 & 14 & 29 \\
\hline 45 & Telamonia dimidiata & 13 & 12 & 10 & 35 \\
\hline 46 & Tetragnatha mandibulata & 9 & 11 & 8 & 28 \\
\hline 47 & Tetragnatha spp.1 & 1 & 2 & 0 & 3 \\
\hline 48 & Thelacantha brevispina & 9 & 9 & 0 & 18 \\
\hline 49. & Theridion spp. & 9 & 10 & 9 & 28 \\
\hline 49 & Thomisus spectabilis & 4 & 5 & 0 & 9 \\
\hline 51 & Uloborus spp & 4 & 5 & 2 & 11 \\
\hline \multicolumn{2}{|l|}{ Total count during each season } & 753 & $\mathbf{7 8 8}$ & & 650 \\
\hline
\end{tabular}

Table.3: Frequency, density, abundance and species importance value of each species

\begin{tabular}{|c|c|c|c|c|c|}
\hline $\begin{array}{c}\text { Sl } \\
\text { No. }\end{array}$ & Species & $\mathbf{F}$ & D & $\mathbf{A}$ & SIV \\
\hline 1 & Araneus spp & 1.00 & 7.67 & 7.67 & 3.21 \\
\hline 2 & Argiope anasuja & 1.00 & 11.33 & 11.33 & 3.76 \\
\hline 3 & Argiope pulchella & 1.00 & 11.67 & 11.67 & 3.71 \\
\hline 4 & Argiope spp.1 & 1.00 & 4.67 & 4.67 & 2.80 \\
\hline 5 & Asemonea spp & 1.00 & 7.67 & 7.67 & 3.21 \\
\hline 6 & Bavia kairali & 0.67 & 1.00 & 1.50 & 1.58 \\
\hline 7 & Carrhotus viduus & 1.00 & 3.67 & 3.67 & 2.66 \\
\hline 8 & Castianeira zetes & 1.00 & 14.00 & 14.00 & 4.08 \\
\hline 9 & Chrysilla volupe & 1.00 & 13.67 & 13.67 & 4.03 \\
\hline 10 & Clubiona spp & 1.00 & 9.33 & 9.33 & 3.44 \\
\hline 11 & Crossopriza lyoni & 1.00 & 51.67 & 51.67 & 9.23 \\
\hline 12 & Cyclosa bifida & 1.00 & 21.00 & 21.00 & 5.03 \\
\hline 13 & Cyrtophora cicatrosa & 1.00 & 36.33 & 36.33 & 7.13 \\
\hline 14 & Cyrtophora citricola & 1.00 & 17.33 & 17.33 & 4.53 \\
\hline 15 & Epeus indicus & 1.00 & 3.67 & 3.67 & 2.66 \\
\hline 16 & Eriovixia spp. & 1.00 & 3.33 & 3.33 & 2.61 \\
\hline 17 & Gasteracantha geminata & 0.67 & 8.00 & 12.00 & 2.53 \\
\hline 18 & Gasteracantha spp.1 & 0.67 & 1.00 & 1.50 & 1.58 \\
\hline 19 & Geolycosa spp. & 1.00 & 45.00 & 45.00 & 8.32 \\
\hline 20 & Gnaphosa spp & 1.00 & 5.67 & 5.67 & 2.93 \\
\hline 21 & Heteropoda venatoria & 1.00 & 4.67 & 4.67 & 2.80 \\
\hline 22 & Hersilia savignyi & 1.00 & 10.67 & 10.67 & 3.62 \\
\hline 23 & Hippasa agelenoides & 1.00 & 30.00 & 30.00 & 6.27 \\
\hline 24 & Hyllus semicupreus & 1.00 & 3.00 & 3.00 & 2.57 \\
\hline 25 & Leucauge decorata & 1.00 & 11.33 & 11.33 & 3.71 \\
\hline 26 & Leucauge tessellata & 0.67 & 2.00 & 3.00 & 1.71 \\
\hline 27 & Leucauge spp.1 & 0.67 & 1.00 & 1.50 & 1.58 \\
\hline 28 & Menemerus bivittatus & 1.00 & 9.00 & 9.00 & 3.39 \\
\hline 29 & Misumenops rubrodecoratus & 0.67 & 1.00 & 1.50 & 1.58 \\
\hline 30 & Myrmarachne plataleoides & 1.00 & 16.00 & 16.00 & 4.35 \\
\hline 31 & Neoscona crucifera & 1.00 & 33.00 & 33.00 & 6.68 \\
\hline 32 & Neoscona nautica & 1.00 & 3.00 & 3.00 & 2.57 \\
\hline 33 & Neriene sundaica & 1.00 & 26.33 & 26.33 & 5.76 \\
\hline
\end{tabular}




\begin{tabular}{|l|l|r|r|r|r|}
\hline 34 & Oxyopes javanus & 1.00 & 48.33 & 48.33 & 8.78 \\
\hline 35 & Oxyopes sunandae & 0.33 & 0.67 & 2.00 & 0.81 \\
\hline 36 & Opadometa fastigata & 0.67 & 1.33 & 2.00 & 1.62 \\
\hline 37 & Parawixia dehaani & 0.67 & 0.67 & 1.00 & 1.53 \\
\hline 38 & Pardosa spp & 1.00 & 53.67 & 53.67 & 9.51 \\
\hline 39 & Peucetia viridans & 1.00 & 20.33 & 20.33 & 4.94 \\
\hline 40 & Phintella vittata & 1.00 & 4.67 & 4.67 & 2.80 \\
\hline 41 & Pholcus spp & 1.00 & 53.33 & 53.33 & 9.46 \\
\hline 42 & Plexippus paykulli & 1.00 & $\mathbf{6 3 . 6 7}$ & $\mathbf{6 3 . 6 7}$ & $\mathbf{1 0 . 8 8}$ \\
\hline 43 & Portia albimana & 0.67 & 1.33 & 2.00 & 1.62 \\
\hline 44 & Stegodyphus sarasinorum & 1.00 & 9.67 & 9.67 & 3.48 \\
\hline 45 & Telamonia dimidiata & 1.00 & 11.67 & 11.67 & 3.76 \\
\hline 46 & Tetragnatha mandibulata & 0.67 & 1.00 & 1.50 & 1.58 \\
\hline 47 & Tetragnatha spp.1 & 1.00 & 9.33 & 9.33 & 3.44 \\
\hline 48 & Thelacantha brevispina & 0.67 & 6.00 & 9.00 & 2.26 \\
\hline 49. & Theridion spp. & 1.00 & 9.33 & 9.33 & 3.44 \\
\hline 49 & Thomisus spectabilis & 0.67 & 3.00 & 4.50 & 1.85 \\
\hline 51 & Uloborus spp & 1.00 & 3.67 & 3.67 & 2.66 \\
\hline
\end{tabular}

F- Frequency; D- Density; A- Abundance; SIV- Species Importance Value

Frequency value is 1 for those species which found throughout all seasons, 0.67 for which found in only two seasons and 0.33 tells it was found only in one season. Highest density is 63.67 for Plexippus paykulli, followed by 53.67 of Pardosa spp. The least density is of Parawixia dehaani which is 0.67. Abundant species was Plexippus paykulli showing the abundant value 63.67 and the least abundant was Parawixia dehaani, having the abundant value 1.00. SIV is highest for Plexippus paykulli i.e. 10.88 shows the greater dominancy of the species followed by Pardosa spp. with 9.51 and least was for Parawixia dehaani.

Shannon index is 3.39 and Simpson value is 0.447 which indicates greater biodiversity of spiders in the study area (Table.3).

\section{CONCLUSION}

From the present study it is evident that spiders can survive in different habitats as Ground runners (Lycosidae, Salticidae, Corinnidae, Gnaphosidae, and Sparassidae) Foliage runners (Clubionidae, Hersiliidae) Stackers (Oxyopidae and Salticidae), Ambushers (Thomisidae), and Web-builders. The types of vegetation greatly affect the population and diversity of spiders. The study area being an urban region, provide a good environment for the survival of spiders. Rainy season and winter season provide favourable condition for these creatures by supplying a large population of prey insects. Shannon index being 3.39 indicates good species diversity in all three main seasons. The individual count was highest during winter season and lowest during summer season. This indicates overall diversity richness in the study area as they are both good predator and prey. Species occurrence and abundance is also related to habitat preference.

\section{ACKNOWLEDGEMENTS}

Authors wish to thank the Head and Chairpersons, Department of Applied Zoology, Kuvempu University, Shimoga, for their encouragement, we would like to thank Sri. Prashantha Kumara SM, Research Scholar, Department of Applied Zoology who helped us in spider identification and species confirmation, we express our gratitude to Sri. Aditya Rao GS, Research Scholar, Department of Applied Botany and Vishwajith U from Department Applied Zoology, for their continuous support in completing this paper, Authors are very grateful to Villagers of Malavagoppa and are acknowledged for their continuous support and cooperation to carry out field surveys at their village.

\section{REFERENCES}

[1] Adarsh, C.K. \& P.O. Nameer. (2015). A preliminary checklist of spiders (Araneae: Arachnida) in Chinnar Wildlife Sanctuary, Western Ghats, India. Journal of Threatened Taxa 8(4): 8703-87

[2] Foelix, R. F. (1996). Biology of Spiders. II edition, Oxford University Press, New York.

[3] Jose, A. C., Sudhin, P. P., Prasad, P. M., Shreejit, K. A. (2018). Spider Diversity in Kavvayi River Basin, 
Kerala, South India. Current World Environment. Vol. 13, No. (1) 2018. ISSN: 0973-4929. Pg 100112.

[4] Kremen, C.,Colwell, R K.,Erwin, T.L., Murphy, D. D., Noss, R. F. and Sanjayan, M. A. (1993). Terrestrial arthropod assemblages: Their use in conservation planning. Conservation biology. 7: 796-808.

[5] Nalini Bai and Ravindranath (2012). Spider diversity in IISc. Bangalore, India. Indian journal of Arachnology.

[6] Patel, M. B. and Patel, M. L. (2018). Web Making Activity in Spiders in Semi-arid Zone. International Research Journal of Biological Sciences. Vol. 7(1), 20-25, Jan 2018. ISSN: 2278-3202.

[7] Prashanthakumara S.M, Nijagal B.S, Venkateshvaralu M. (2015). Study on diversity of spider fauna in Jnana Sahyadri campus, Shimoga, Karnataka. Bulletin of pure and applied zoology. Vol. 34 A. Issue (No 1-2): P.1-7

[8] Prashanthakumara, S. M. and Venkateshwarlu, M. (2017). Diversity and distribution of spider fauna in different ecosystems of Chikkamagaluru parts of Western Ghats, Karnataka. International journal of innovative research and advanced studies (IJIRAS). Volume-4, issue-7.

[9] Prashathakumara, S. M. and Venkateshwarlu M. (2017). Preliminary study of spiders (Araenae) in Gudavi Bird Sanctury, Shimoga, Karnataka. International journal of recent scientific research volume 8, is sue 8, pp 19277-19281, 2017.

[10] Riechert, S.E. and T. Lockley. 1984. Spiders as biol ogical control agents. Annual Review of Entomolog y 29: 299- 320 .

[11] Sebestian, P. A. and Peter, K. V. (2009). Spiders of India, Universities Press/Oriennt Blackswan.

[12] Sebestian, P. A. and Peter, K. V. (2010). Spiders of India, Universities Press (India) Pvt. Ltd.
[13] Sebestian, P. A., Sudhikumar, A. V., Mathew, m. J. and Sunish, E. Diversity of spiders (Aranae) in the Western Ghats-an overview. 2012.

[14] Shirbhate, M. V. and Shirbhate. (2017). Diversity and Distribution of Spider Fauna (Family: Araneidae) in and around Katepurna Sanctuary, Akola, India. Environment Conservation Journal18 (3) 45-52. ISSN: 0972-3099 (print) 22785124(online).

[15] Sumangala Rao, Shrikanth, Shreya K., Ahwini V. Rekha K. N. and Shenoy K. B. (2018). Spider diversity on Mangaluru University campus. Journal of Entomology and Zoology Studies; 6(2):31883194.

[16] Tikader, B. K. (1960). On some new species of spiders (Arachnida) of the family Thomisidae from India. BNHS. 284-288.

[17] Tikader, B. K. (1987). Handbook of Indian Spiders: A Manual for the study of the Spiders and Their Relatives: the Scorpions, Pseudoscorpions, Whip Scorpions, Harvestmen and all members of class Arachnida Found in India, with Analytical keys for their classification and Biology. Zoological Survey of India, Calcutta, India.

[18] Upamanyu Hore and V.P. Uniyal, (2008). Diversity and composition of spider assemblages in five vegetation types of the Terai Conservation Area, India. Journal of Arachnology, 36(2):251-258/'p/

[19] Vijayakumar. (2002). Status of paddy insect pests and their natural enemies in Tungabhadra project area, MSc Thesis, University of Agricultural Sciences, Dharwad, Karnataka (India).

[20] World Spider Catalog (2019). World Spider Catalog. Version 20.0. Natural History Museum Bern, Online at http://wsc.nmbe.ch, accessed on 10.04.2019. doi: 10.24436 .

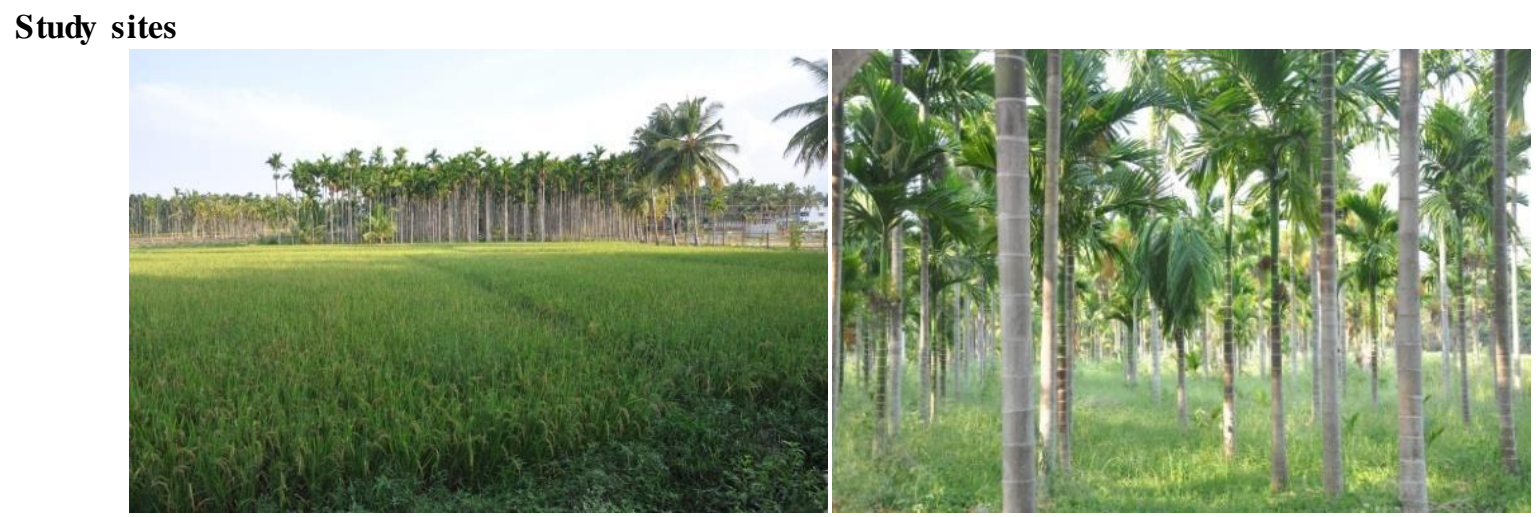



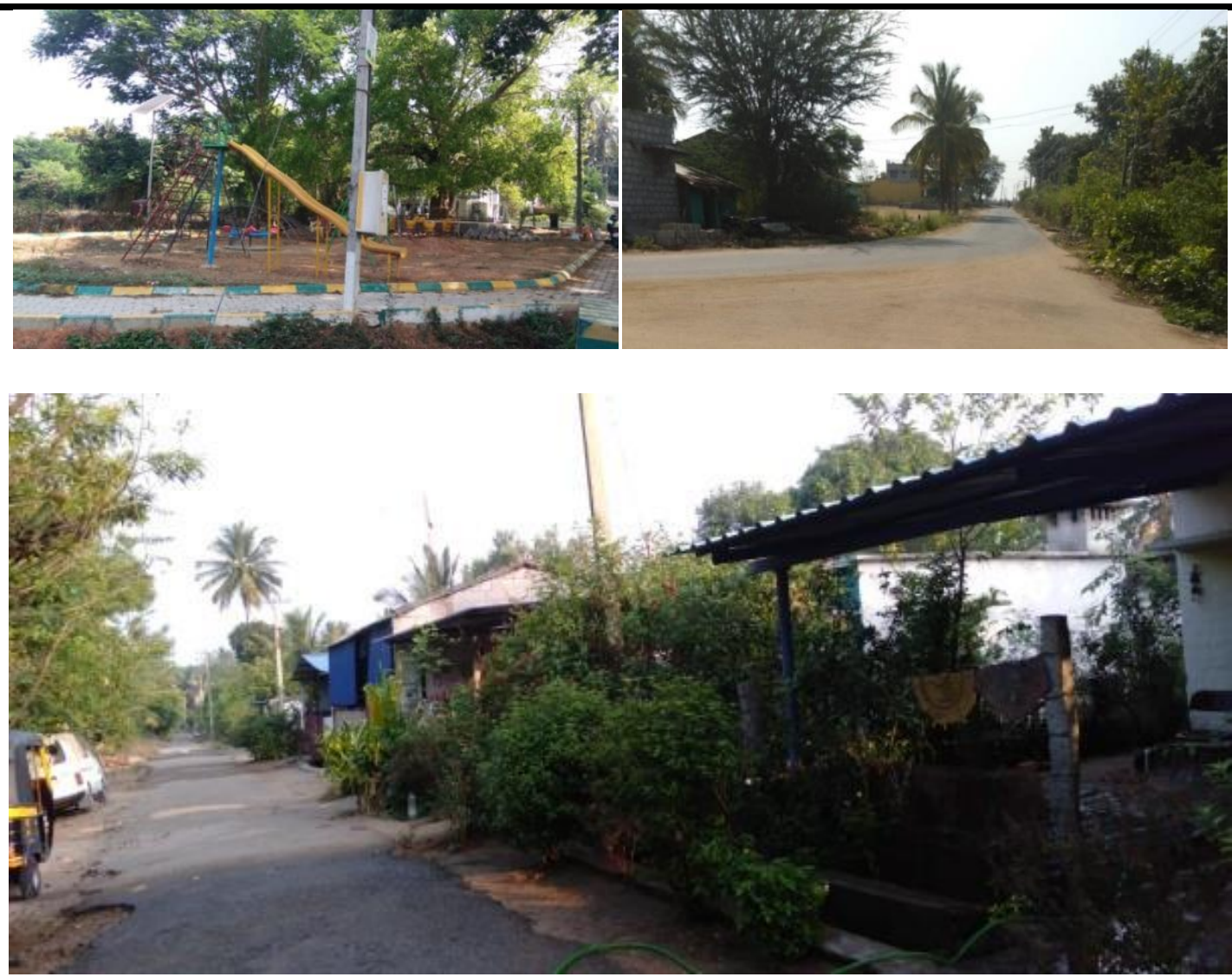

Species Plates

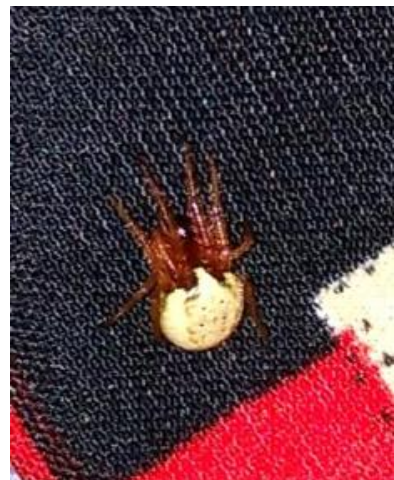

Araneus spp

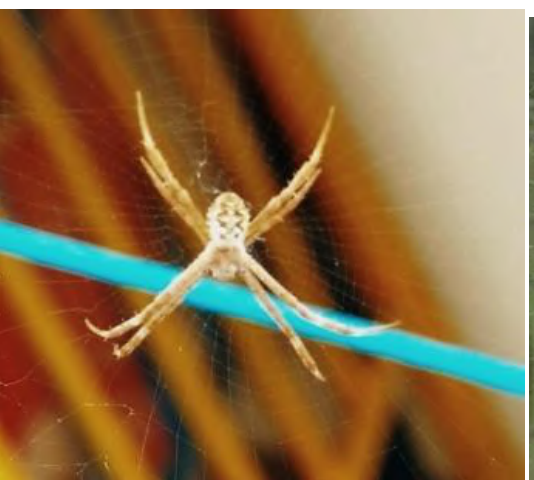

Argiope spp.1

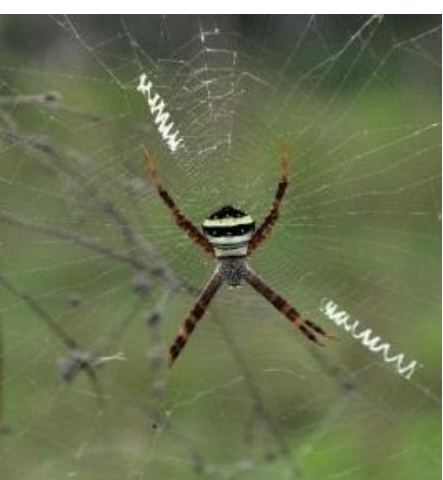

Argiope pulchella

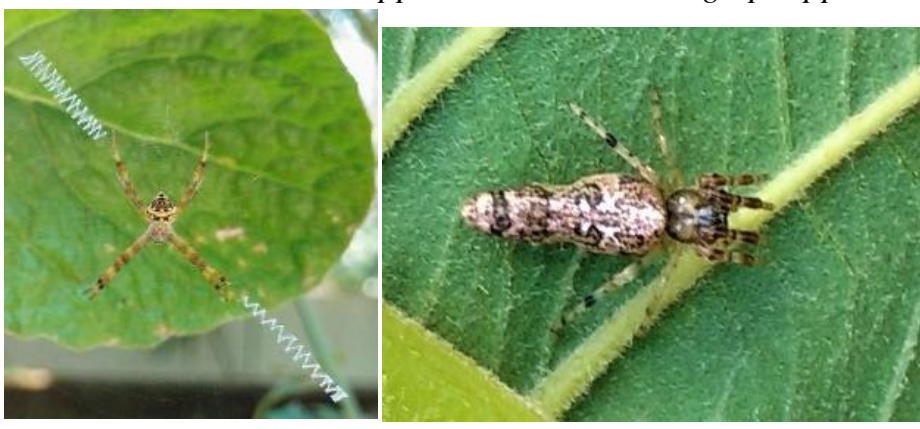

Argiope anasuja
Cyclosa bifida

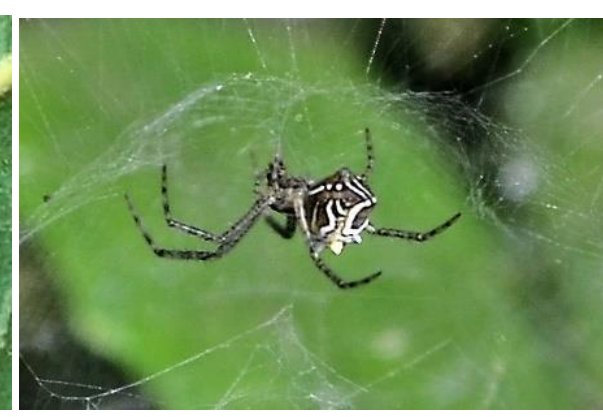

Cyrtophora citricola 


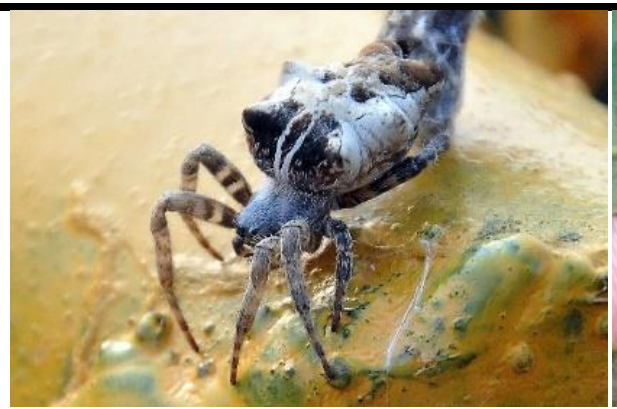

Cyrtophora citricola

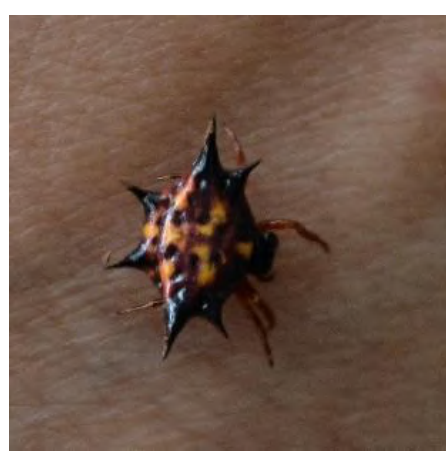

Gasteracantha spp.1

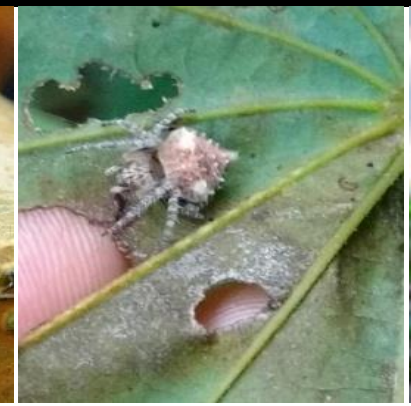

Eriovixia spp

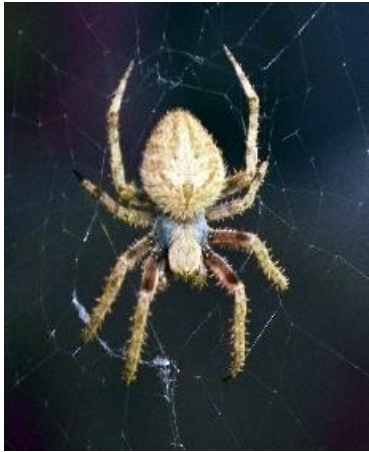

Neoscona crucifera

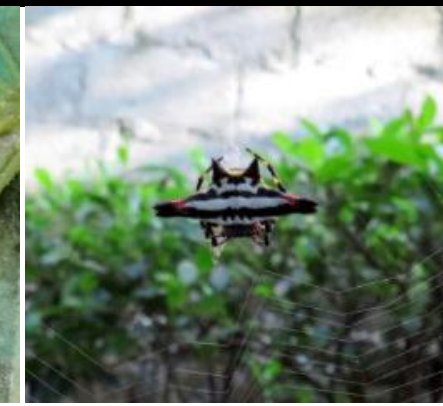

Gasteracantha geminata

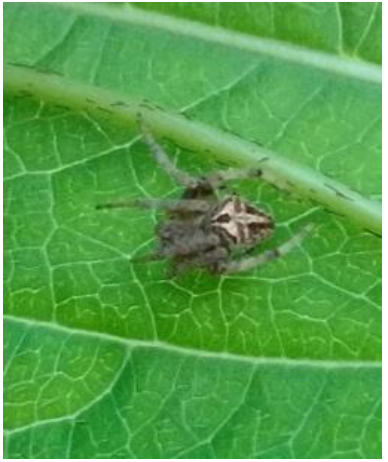

Neoscona nautica

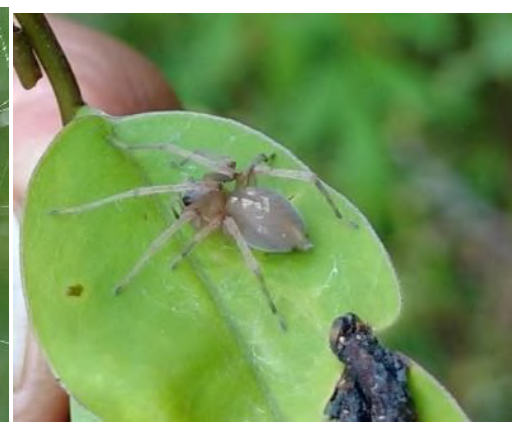

Clubiona spp

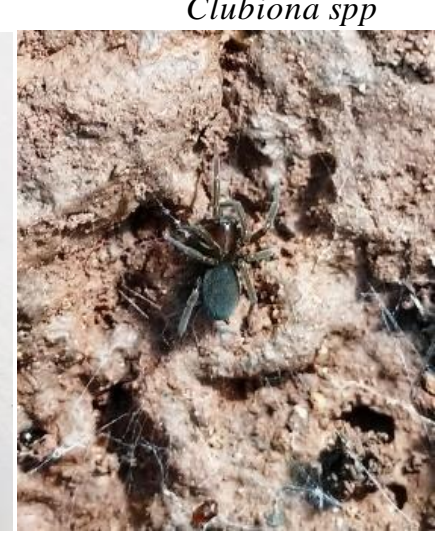

Gnaphosa spp.

Thelacantha brevispina

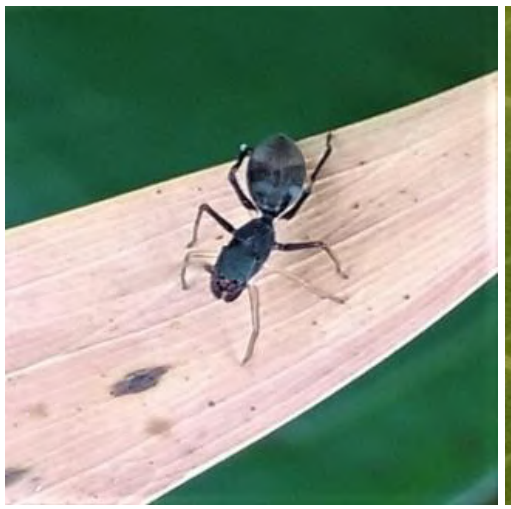

Castianeira zetes

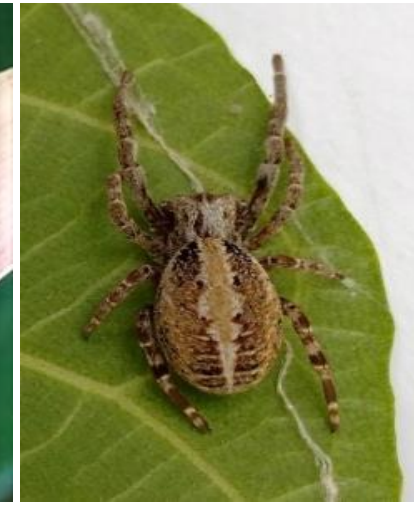

Stegodyphus sarasinorum 


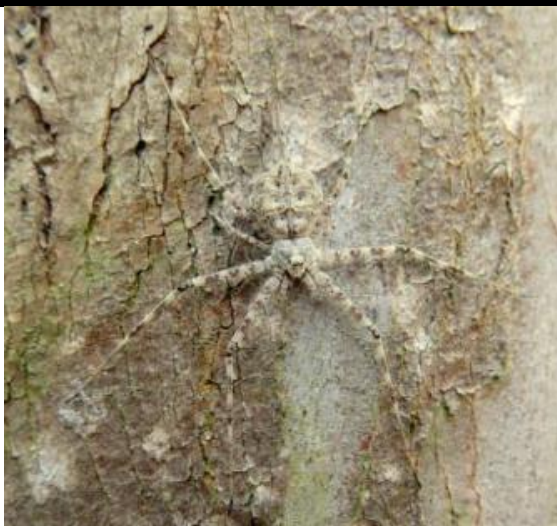

Hersilia savignyi

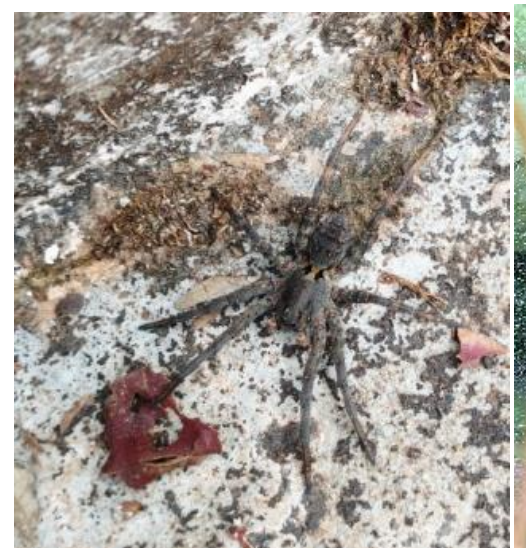

Geolycosa spp

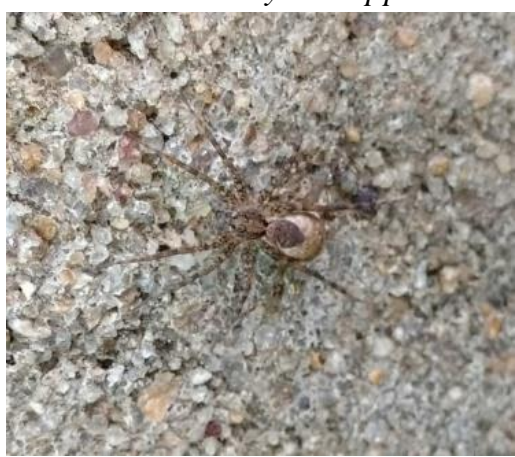

P ardosa spp.

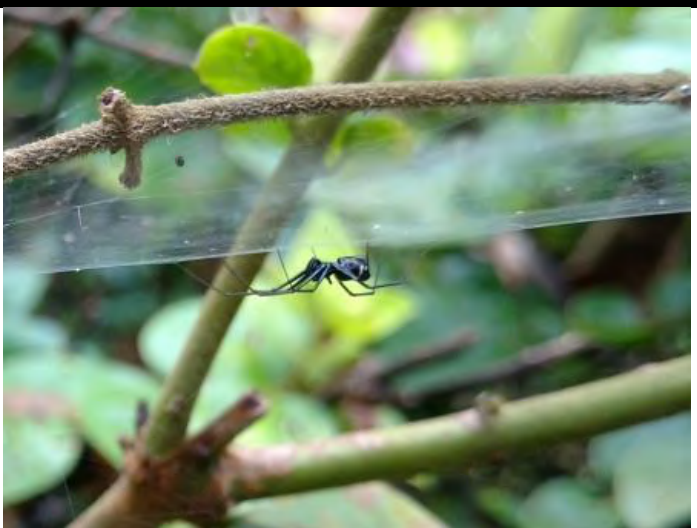

Neriene sundaica

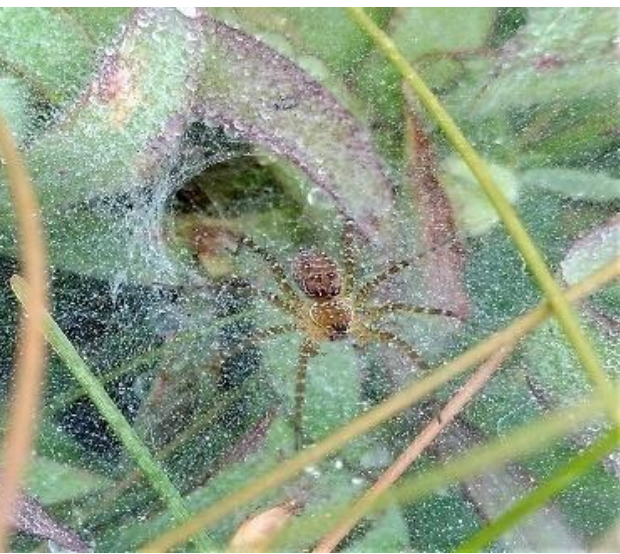

Hippasa agelenoides

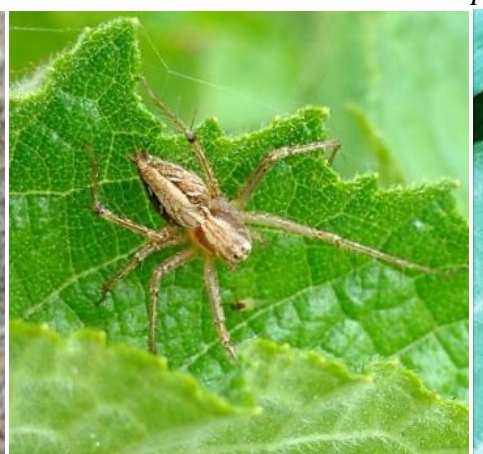

Oxyopes javanus

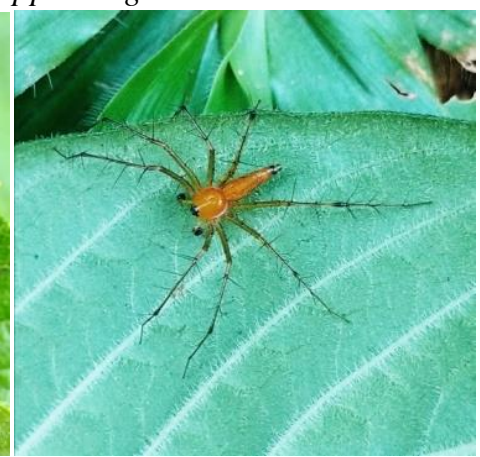

Oxyopes sunandae

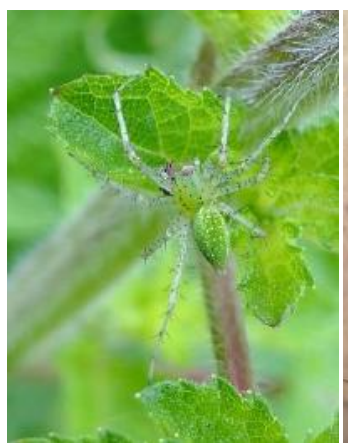

Peucetia viridians

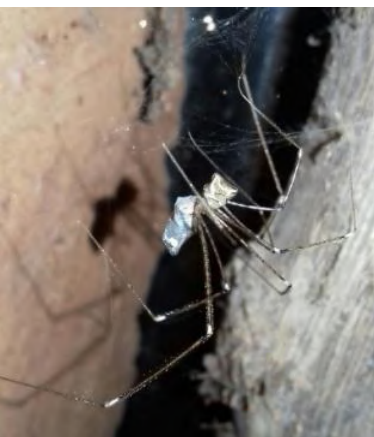

Crossopriza lyoni

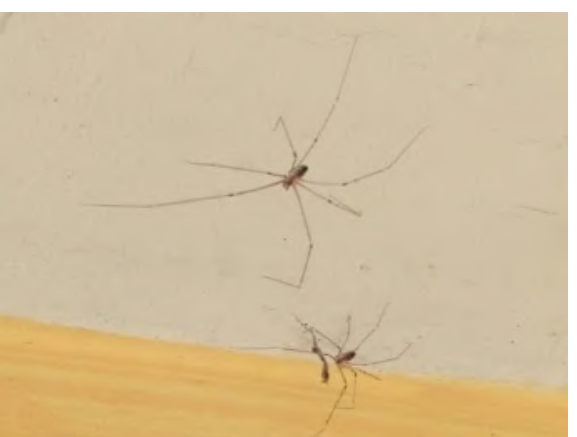

Pholcus spp. 


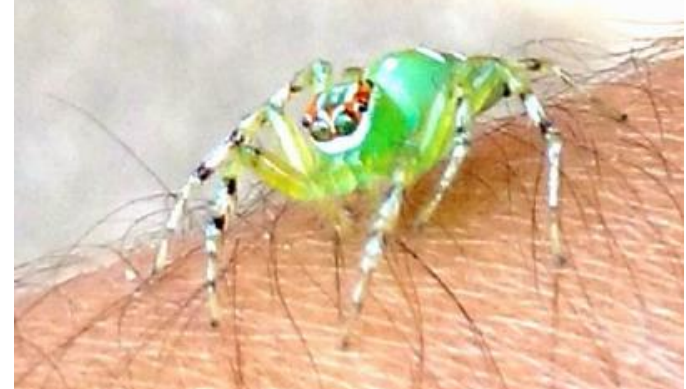

Asemonea spp

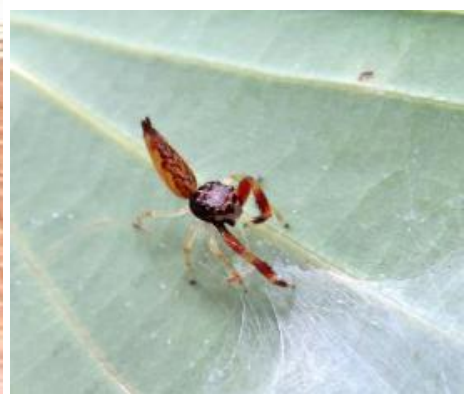

Bavia kairali

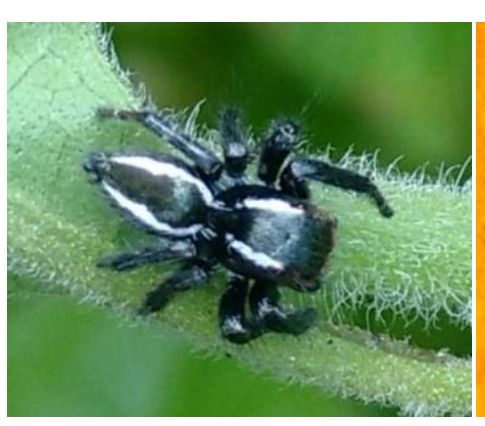

Carrhotus viduus

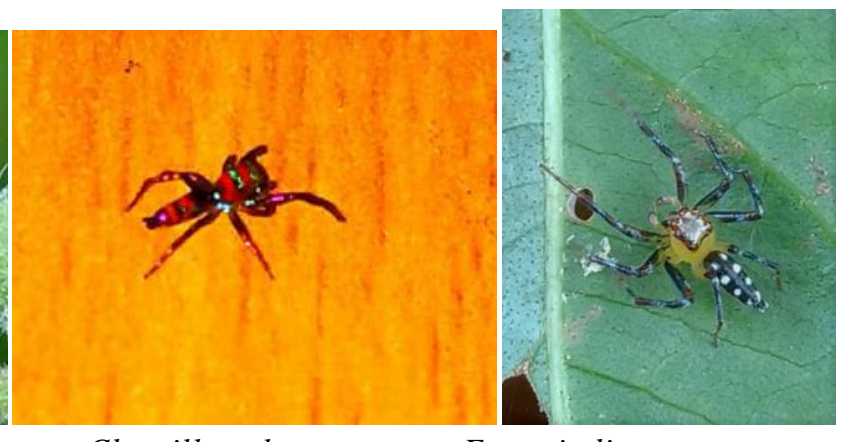

Chrysilla volupe

Epeus indicus

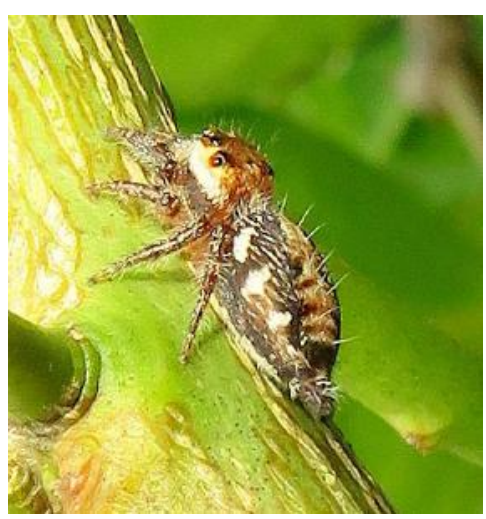

Hyllus semicupreus

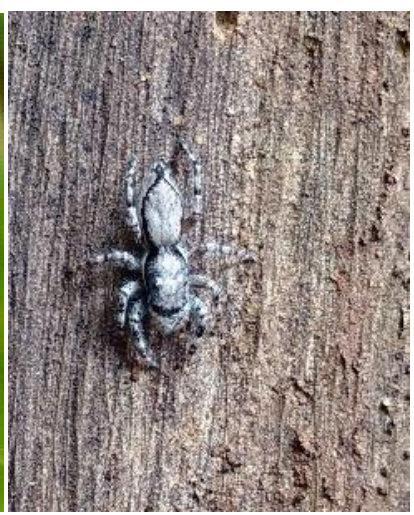

Menemerus bivittatus

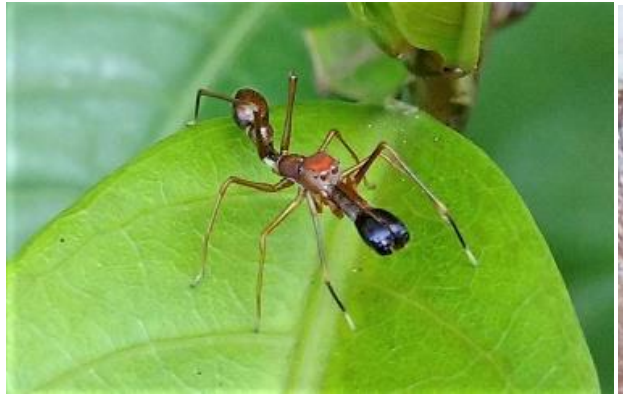

Myrmarachne plataleoides

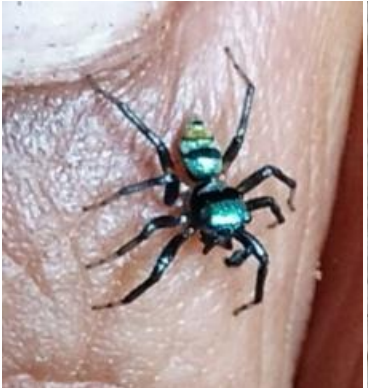

Phintella vittata

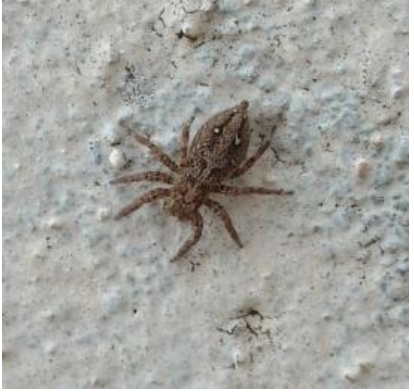

Plexippus paykulli 


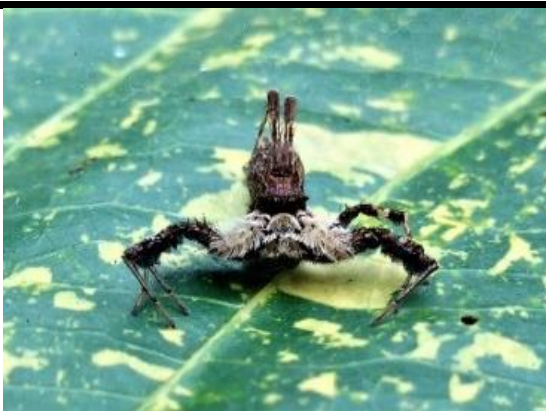

Portia albimana

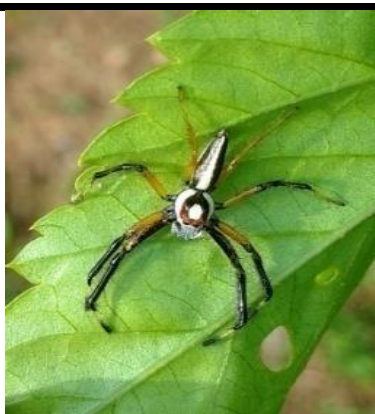

Telamonia dimidiata

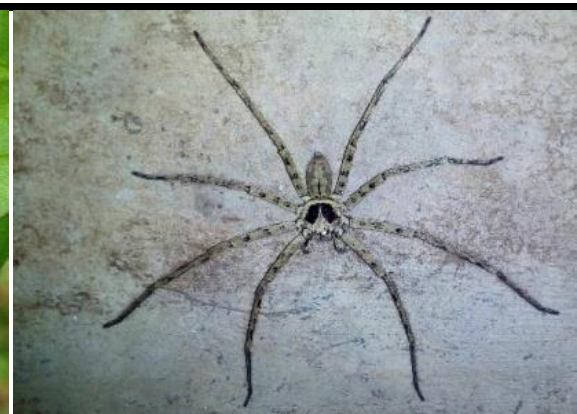

Heteropoda venatoria

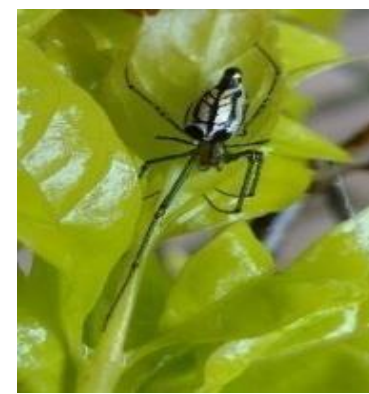

Leucauge decorata

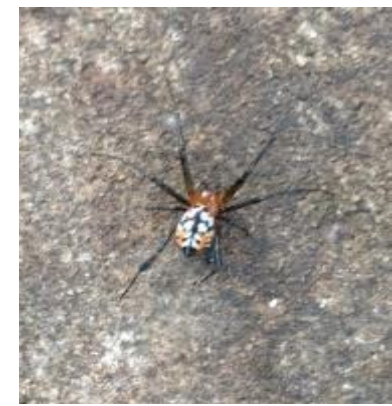

Opadometa fastigata

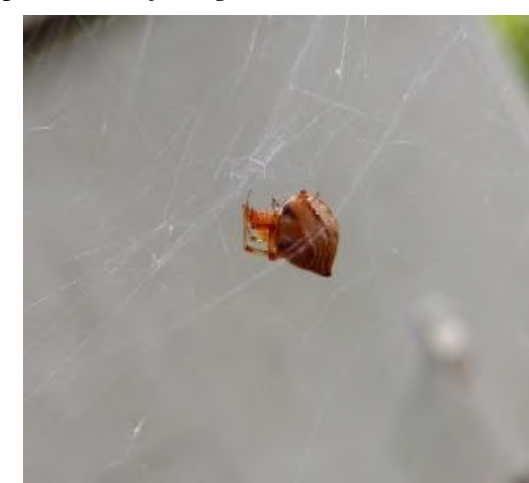

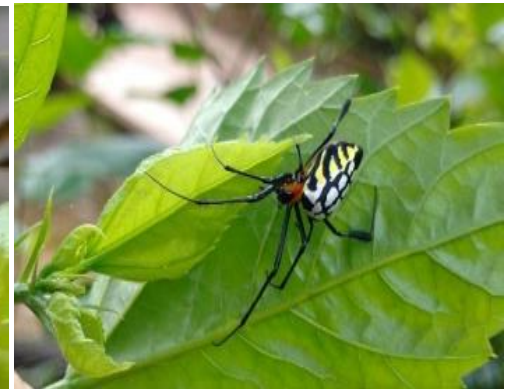

Leucauge tessellata

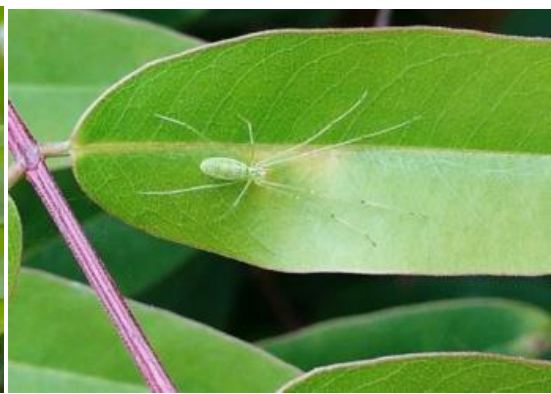

Leucauge spp.1

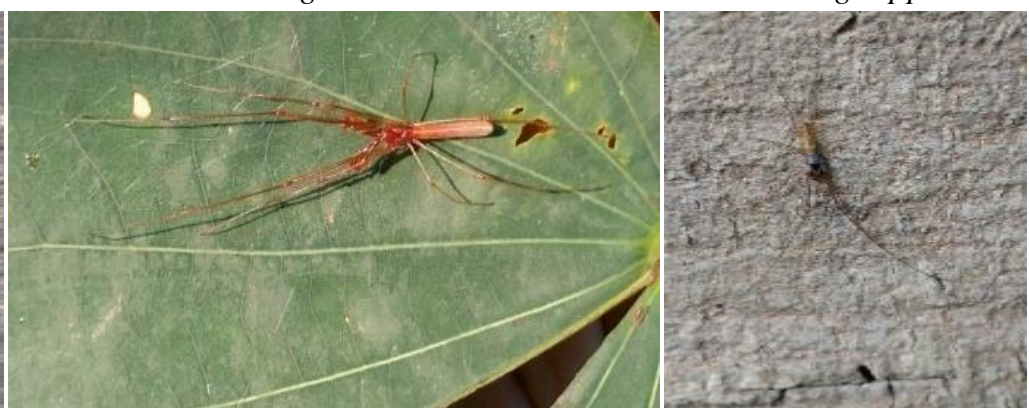

Tetragnatha spp.1

Tetragnatha mandibulata
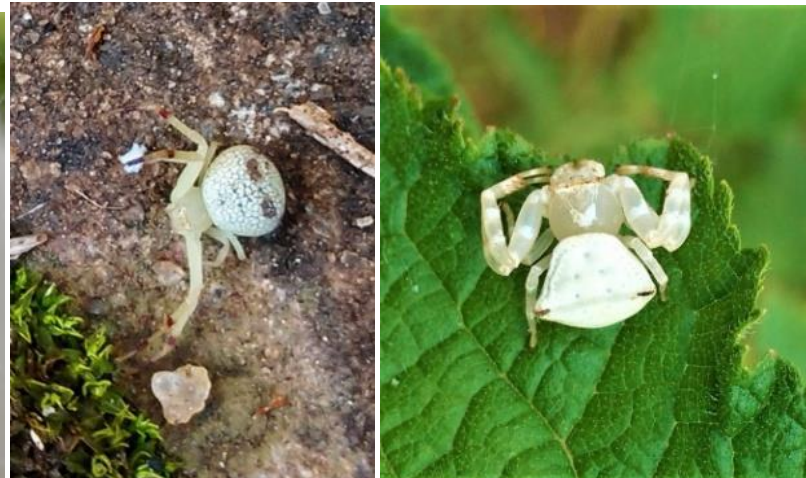

Theridion $s p p$

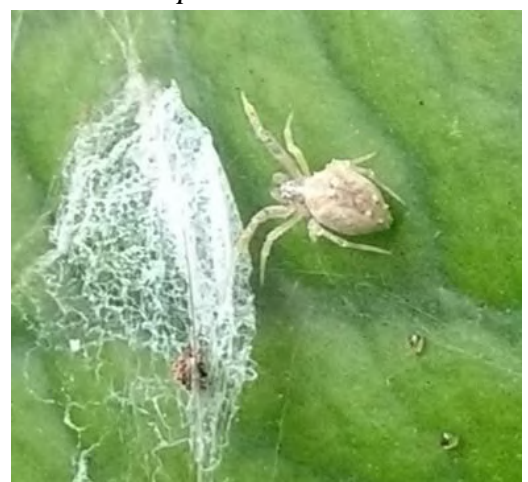

Uloborus spp 